\title{
Using artificial neural network models for the prediction of thrust force and torque in drilling operation of Al7075
}

\author{
Panagiotis Kyratsis ${ }^{1 *}$, Nikolaos Efkolidis ${ }^{1}$, Daniel Ghiculescu ${ }^{2}$ and Konstantinos Kakoulis ${ }^{1}$ \\ ${ }^{1}$ Dept. of Mechanical Engineering \& Industrial Design of the Western Macedonia University of \\ Applied Sciences, Kila Kozani, GR50100, Greece \\ ${ }^{2}$ Dept. of Manufacturing Technology, University Politehnica of Bucharest, Bucharest, 313 Splaiul \\ Independentei, Romania
}

\begin{abstract}
This study investigates the thrust force $(\mathrm{Fz})$ and torque $(\mathrm{Mz})$ in a drilling process of an A17075 workpiece using solid carbide tools (Kennametal KC7325), depending on the effects of crucial cutting parameters such as cutting velocity, feed rate and tool diameter of $10 \mathrm{~mm}$, $12 \mathrm{~mm}$ and $14 \mathrm{~mm}$. Artificial neural networks (ANN) methodology is used in order to acquire mathematical models for both the thrust force $(\mathrm{Fz})$ and torque $(\mathrm{Mz})$ related to the drilling process. The ANN results showed that the best prediction topology of the network for the thrust force was the one with five neurons in the hidden layer, while for the case of $\mathrm{Mz}$ the best network topology for the prediction of the experimental values was the one with six neurons in the hidden layer. Based on the results acquired, the ANN models achieved accuracy of $1,96 \%$ and $1,95 \%$ for both the thrust force and torque measured, while the R coefficient for the prediction model of the thrust force is 0.99976 and 00.99981 for the torque. As a result they can be considered as very accurate and appropriate for their prediction.
\end{abstract}

\section{Introduction}

Aluminum alloy (A17075) is a suitable material for a variety of specific applications in aeronautical and aerospace sectors. Its mechanical and thermal properties such as high strength comparable to many steels, low density, good thermal conductivity, make it very popular for highly stressed structural parts. As proper material for high tech engineering applications, has made a lot of researchers to focus their research discovering its manufacturing attitude and its characteristics [1-5].

Artificial Neural Networks can be successfully applied to many industrial situations. Predictive neural network models were found to be better at predicting many crucial parameters through many manufacturing processes. This study focuses on the drilling process. Tahavvor et al [6] investigated the relation between the temperature of drilling hole during the drilling process and the workpiece quality and tool life. They used Artificial Neural Networks for the determination of the temperature of the drilling hole according to

${ }^{*}$ Corresponding author: pkyratsis@teiwm.gr 
certain points' temperature of the workpiece and two parameters, drill diameter and ambient temperature. Vrabel et al [7] developed an artificial neural network for the prediction of the surface roughness of a nickel based super alloy (Udimet 720). Cutting conditions, selected monitoring indices and tool wear parameter were given as the crucial factors of the study. Nieslony et al [8] investigated drilling process of titanium plated steel using two types of indexable insert drills with different configuration of the tool coatings (TiAlN/AlTiN + TiN and TiAlN/TiN). Surface morphology of the drilled holes and contact area was analysed by the analysis of thrust force, torque and signal fluctuations of PSD function. Manufacturing properties of materials and surface roughness are factors which influence the running conditions.

Beruvides et al [9] explored the thrust force and perpendicular vibrations in micro drilling processes of five commonly used alloys (titanium-based, tungsten-based, aluminum-based and invar). They developed two different models based on artificial neural networks and on fuzzy inference systems. The study was related on peck drilling and the influence of five cutting parameters. Patra et al [10] investigated the tool wear of the micro drill and thrust force during the peck drilling operation of AISI P20 tool on a steel workpiece. Artificial neural network model was developed to fuse thrust force, cutting speed, spindle speed and feed parameters to predict the drilled hole number. Qin et al [11] explored the better hole quality with one-time machining by helical milling in a carbon fiber-reinforced plastic. A neural network model developed based on the correlation between the delamination and the process parameters. The effects of the process parameters on delamination at the exit of the machined holes were analyzed by using this model and the predicted results. Leite et al [12] presented an experimental methodology of Design for Manufacturing, used for survey and analysis of geometric deviations of CNC MachineTools, through their final product. They developed an Artificial Neural Network to predict the fabrication parameters in order to avoid costs, which are generated by these deviations. Yildırım et al [13] developed a neural network-based approach for plastic material and different feed rates under different working speeds, in order to examine some vibrations and heating problems of drill machines during drilling process. According to the research the model can be used as predictor of those problems in real time applications during drilling process. The aforementioned research works proved that ANN is a methodology that can be used effectively for a variety of tasks and models. The current paper focuses and modelling the thrust force and torque in drilling A17075 and provides evidence of accuracy of the proposed models.

\section{Experimental settings}

In this research, an A17075 block was used as a workpiece material. The dimensions of this workpiece material were $150 \mathrm{~mm} \times 150 \mathrm{~mm} \times 15 \mathrm{~mm}$. For the drilling tests, a HAAS VF1 CNC machining center with continuous speed and feed control was used. During the tests, three cutting speeds ( $\mathrm{V}-10,40$ and $70 \mathrm{~m} / \mathrm{min}$ ) and three feed rates ( $\mathrm{f}-0.2,0.4$ and $0.6 \mathrm{~mm} / \mathrm{rev}$ ) were selected to be applied with three different cutting tools diameters (D - 10, 12 and $14 \mathrm{~mm})$. Thrust force $(\mathrm{Fz})$ and torque $(\mathrm{Mz})$ values in each case were measured by a Kistler dynamometer type 9123 (capable of measuring four components at the same time). A total number of 27 experiments were executed by using all the combinations of cutting speeds, feed rates and cutting tool diameters. Figure 1 depicts the workflow conducted.

The cutting parameters, units and notations are given in Table 1. 


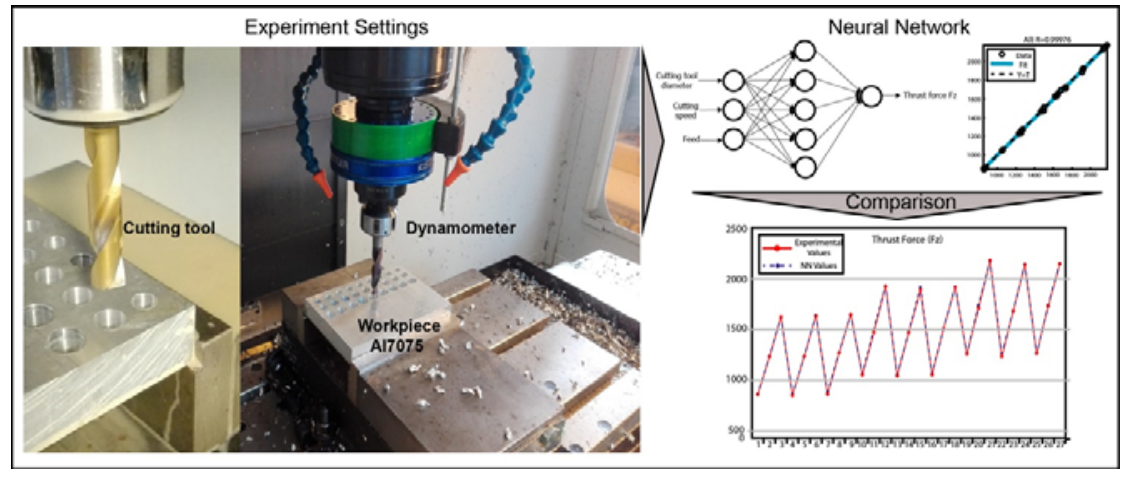

Fig. 1. The workflow of the experiments.

Table 1. Cutting variables used in the experiments.

\begin{tabular}{|c|c|}
\hline Parameters & Values \\
\hline Cutting velocity $(\mathrm{m} / \mathrm{min})$ - V & $10,40,70$ \\
\hline Feed rate $(\mathrm{mm} / \mathrm{rev})$ - f & $0.2,0.4,0.6$ \\
\hline Tool diameter $(\mathrm{mm})$ - D & $10,12,14$ \\
\hline Axial depth of cut $(\mathrm{mm})$ - ap & 15 \\
\hline Workpiece dimension $(\mathrm{mm})$ & $150 \times 150 \times 15$ \\
\hline
\end{tabular}

\subsection{Prediction of thrust forces and torques with ANNs}

Artificial Neural Networks are very suitable for modelling various manufacturing processes because of their ability to learn complex non-linear and multivariable relationships between process parameters. The ANN is a mathematical model which is inspired by the structure and/or functional aspects of the biological neural networks. It is an adaptive system that changes its structure as it is consisted of neurons, which arranged in different layers and linked through variable weights. These weights are calculated through an iterative method, during the training process. ANNs are suitable for modeling complex relationships between inputs and outputs or to find patterns in data. The back-propagation training algorithms, the scaled conjugate gradient (SCG) and Levenberg-Marquardt (LM), were used for ANNs training.

A set of inputs and desired outputs are required to train ANNs. To reduce the time and cost, while obtaining comprehensive knowledge about the thrust force (Fz) and torque (Mz) related to the drilling process over the selected range of parameters, a properly planned experiment is necessary. All the experimental data (27) were divided into three data sets including training, validation and testing. The $70 \%$ of the data were used randomly to build the network, $15 \%$ to measure network generalization and $15 \%$ as a testing set of the neural network. After a number of trials, the best network architectures for the prediction of thrust force was the 3-5-1 topology, while for the case of torque was the 3-6-1 topology. Both network architectures (Figure 2) were consisted from 3 layers. Three inputs were necessary (tool diameter, cutting speed, feed rate), one hidden layer (with the size of $5 \& 6$ respectively) and two outputs (thrust force and torque).

The dashed line of the plots equals to the perfect (result - outputs $=$ targets). The solid line represents the best fit linear regression line between outputs and targets. The $\mathrm{R}$ value is an indication of the relationship between the outputs and targets. As far as R equals to 1 then there is a precise relationship between outputs and targets. If $\mathrm{R}$ is far away less than 1 , then the quality of the model is poor. 

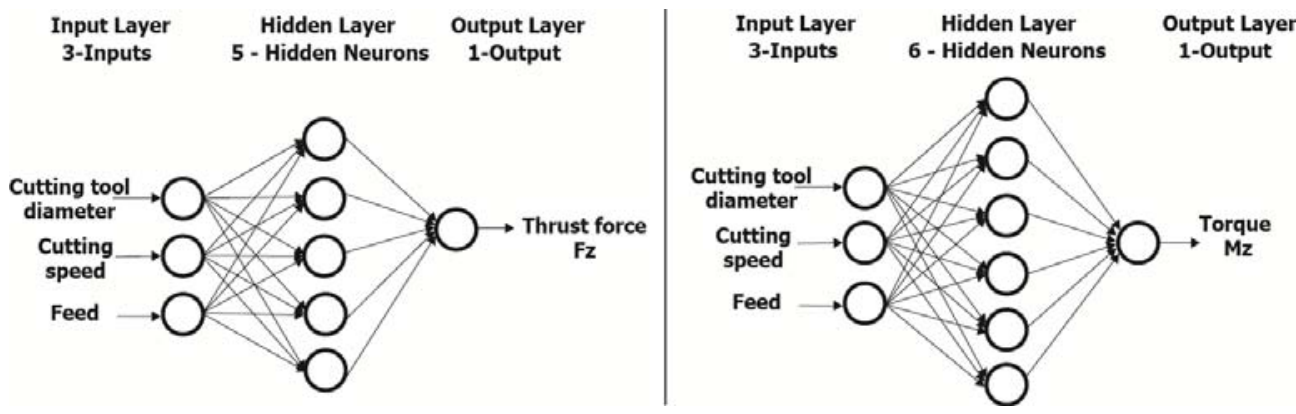

Fig. 2 Network architectures for Fz and Mz.

The plot regression results are shown in the figure below. For the case of the thrust force the figure illustrates the correlation value of the training samples $(\mathrm{R}=0.99999)$, validation samples $(R=0.99988)$, testing samples $(R=0.99995)$, and the fitting correlation of the developed prediction model $(\mathrm{R}=0.99976)$. In the case of the torque correspondingly, the correlation values were for the training samples $\mathrm{R}=0.99997$, for the validation samples $\mathrm{R}=0.99995$, for the testing samples $\mathrm{R}=0.99799$, and finally for the fitting correlation of the developed prediction model equalled to $\mathrm{R}=0.99981$.

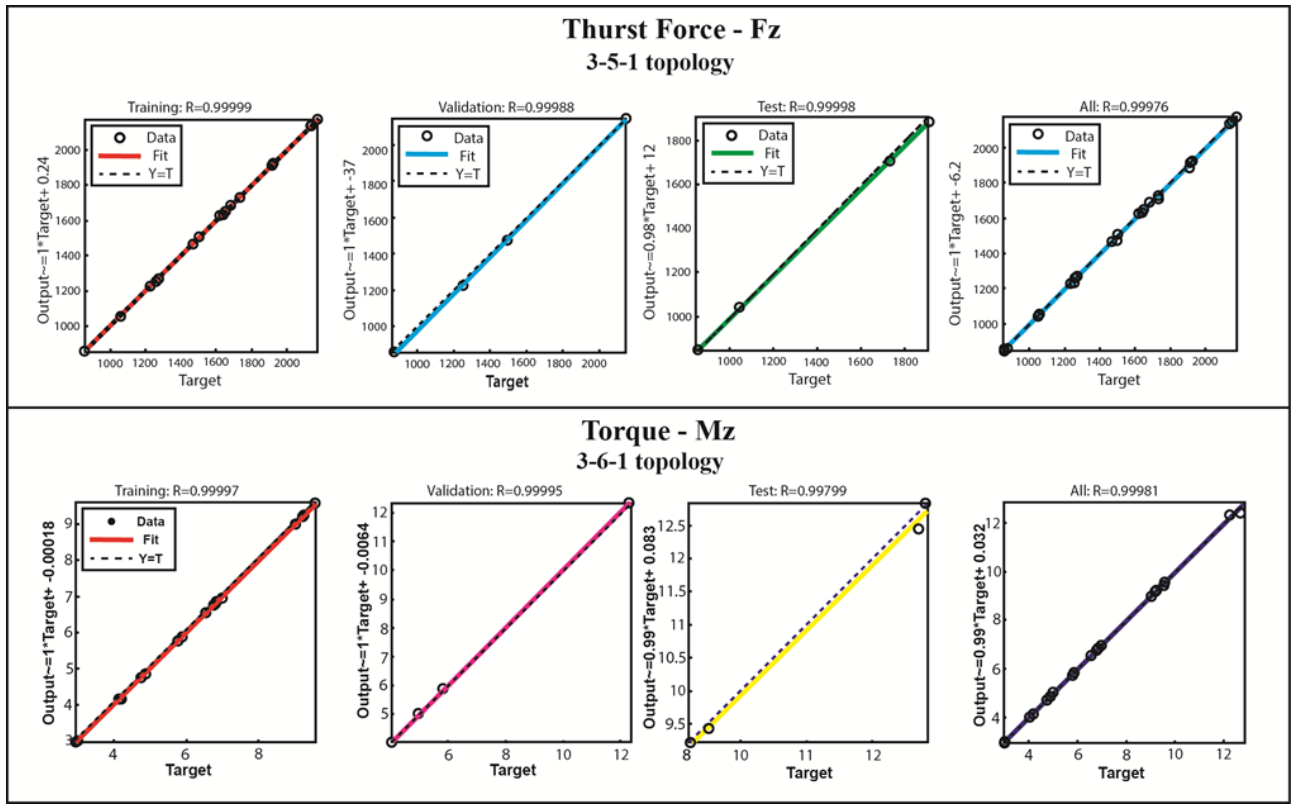

Fig. 3. Regression plot and correlation value $(\mathrm{R})$ of the training, validation, and testing samples.

\subsection{Comparison of experimental vs predicted values}

The comparison between the predicted values of ANN model with the experimental values (Figure 4) shows that in all the cases studied the predicted and experimental values are very close to each other. It is clearly observed, that there is a very good correlation between them. For the prediction model of thrust force, the minimum and the maximum errors were $0.001 \%$ and $1.964 \%$ respectively, while for the case of torque the minimum and the maximum errors were $0.01 \%$ and $1.951 \%$ correspondingly. It is obvious that the neural 
network is a successful tool for predicting the values of $\mathrm{Fz}$ and $\mathrm{Mz}$ based on the experimentally measured ones.
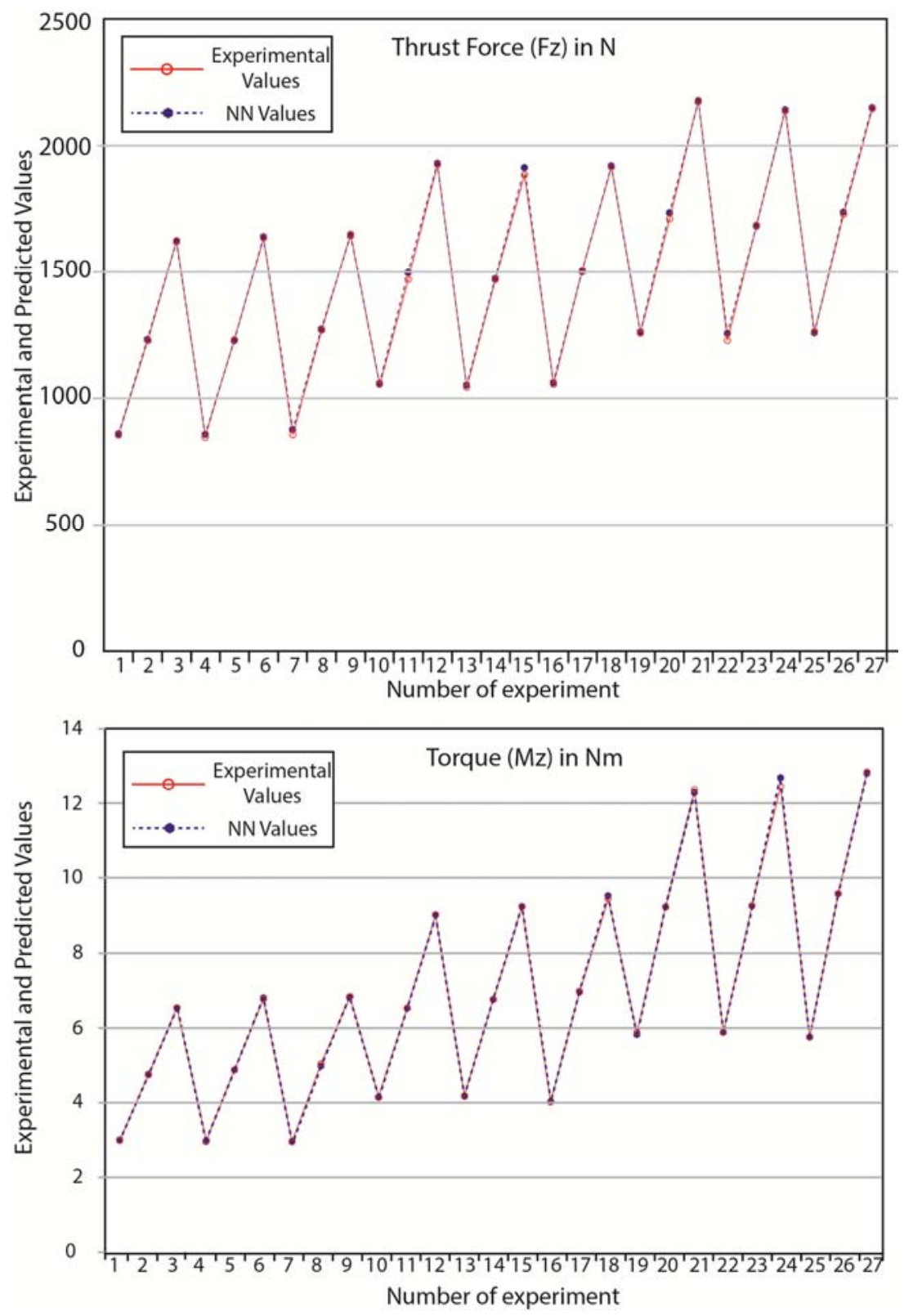

Fig. 4. Presentation of Experimental vs Predicted values.

\section{Conclusions}

In this study, separate mathematical models for the prediction of the thrust force $(\mathrm{Fz})$ and the torque $(\mathrm{Mz})$ developed during the drilling process of an Al7075 workpiece were generated. Basic parameters such as feed rate and cutting speed related to the cutting tools diameter were used in order to develop mathematical models based on artificial neural 
networks. The training sets used $70 \%$ of the data to build the networks, $15 \%$ to measure networks generalization and $15 \%$ as a testing set of the neural networks. The predicted values for the $\mathrm{Fz}$ and $\mathrm{Mz}$ can be considered very accurate because the predicted values were found to be close to those of the experimental work. The best results were obtained using network with five and six hidden neurons for the thrust force and torque respectively. The outcomes proved that when using the ANN models, the accuracy achieved was $1.96 \%$ and $1.95 \%$ for both the thrust force and torque measured. The method can be used in combination with other methodologies in order to highlight its high accuracy. As a result, the usage of ANNs is highly recommended for the prediction of the thrust force and torque during the drilling processes, in order to avoid complex and time-consuming experimental studies.

The authors would like to thank the $\mathrm{C} 3$ (CAD/CAM/CAE) Laboratory Team in the University of Applied Sciences of Western Macedonia, for its valuable help, making available human power and accessible the technical resources.

\section{References}

1. E. K1lıçkap, Int. J. Adv. Manuf. Technol. 49, 911 (2010)

2. İ Ucun, J. Mech. Sci. Technol. 30,1843. (2016)

3. S.B.N Tareg, G. Krallics, Acta Polytech. Hung. 11, 103 (2014)

4. R. Kumar, S. Chauhan, Measurement 65, 166 (2015)

5. V.R. Suryaa, K.M. Vinay, Keshavamurthya, G. Ugrasenb, H.V. Ravindrac, ICMPC2017. 4, 203 (2017)

6. A. Tahavvor, S. Sepehrinia Iran. J Sci. Technol. 38, 269, (2014)

7. M. Vrabel, I. Maňková, J. Beňo, ICPM 2013. 581, 366, (2014)

8. P. Nieslony, P. Cichosz, G.M. Krolczyk, S. Legutko, D. Smyczek, M. Kolodziej. Measurement, 78, 137, (2016)

9. G. Beruvides, F. Castano, R. Haber, R. Quiza, M. Rivas, ICTAI 14. 506 (2014)

10. A.K, Patra, T. Jha, Szalay, J. Ranjan, L. Monostori, Precis. Eng. 48, 279 (2017)

11. X. Qin, B. Wang, B. Wang, H. Li,Y. Jiang, X. Zhang, J. Mech. Sci. Technol. 28, 713 (2014)

12. W.O. Leite, J.C.C, Rubio, J.G. Duduch, P.E.M. Almeida, J. Appl. Soft Comput. 36, 114 (2015)

13. Ş. Yıldırım, E. Esim, Mech. Mach. Sci. 52, (2018) 